\section{A incorporação da avaliação da atenção básica no nível estadual do Sistema Único de Saúde através de processos participativos de ensino-aprendizagem- trabalho}

\section{The inclusion of primary health care evaluation at the State level of the Brazilian National Health System by way of participatory teaching, learning and working practices}

Edson Mamoru Tamaki 1

Ana Rita Barbieri 2

Luiza Helena de Oliveira Cazola 3

Sonia Maria Oliveira de Andrade 4

Oswaldo Yoshimi Tanaka 5

1,3,4 Departamento de Saúde Coletiva. Centro de Ciências Biológicas e da Saúde. Universidade Federal de Mato Grosso do Sul. Cidade Universitária s.n. Campo Grande, MS, Brasil. CEP.79.070-970.E-mail: mamoru@nin.ufms.br

2 Departamento de Enfermagem. Centro de Ciências Biológicas e da Saúde. Universidade Federal de Mato Grosso do Sul. Campo Grande, MS, Brasil.

5 Departamento de Prática de Saúde Pública. Faculdade de Saúde Pública. Universidade de São Paulo. São Paulo, SP, Brasil.

\begin{abstract}
Objectives: to develop an intervention methodology and apply it to the State Health Department teams, as a way of introducing monitoring and evaluation of primary health care by way of training and carrying out a real health planning activity.

Methods: learning and working practices were developed that included training and a health planning activity. The quality of both of these processes was ensured by establishing the principles and criteria for organizing training, drawing up a monitoring and evaluation methodology proposal and teaching practices

Results: the methodology was applied in the Brazilian States of Mato Grosso do Sul (MS), Tocantins (TO) and Amazonas (AM), paying due respect to the specific features of each locality's State Health Department and the qualifications and training of technical staff responsible for primary health care. Methodological proposals were drawn up that were structurally similar but had differing priorities and development proposals. Two years after conclusion of this intervention, the effects could still be identified.

Conclusions: the results obtained in MS, TO and $A M$ reveal the capacity of an approach based on teaching, learning and work to introduce new working practices in health services throughout the institution. Key words Health services evaluation, Primary health care, Institutionalization
\end{abstract}

\section{Resumo}

Objetivos: desenvolver uma metodologia de intervenção e aplicá-la às equipes das Secretarias Estaduais de Saúde (SES) objetivando incorporar a prática de monitoramento e avaliação da atenção básica através de processo de capacitação e realização de atividade real de planejamento e programação em saúde. aprendizagem-trabalho que incorporou ao processo de capacitação uma atividade de planejamento em saúde. A qualidade dos dois processos é assegurada através do estabelecimento de princípios e critérios ração da proposta metodológica de monitoramento e avaliação e para a condução didático-pedagógica do curso

Resultados: a metodologia foi aplicada nos Estados de Mato Grosso do Sul (MS), Tocantins (TO) e Amazonas (AM), respeitando as particularidades locais em termos de organização da SES e da qualificação e capacidade dos técnicos responsáveis pela atenção básica. Foram produzidas propostas metodológicas estruturalmente semelhantes, mas diferentes nas suas prioridades e propostas de desenvolvimento. Dois anos após o término da intervenção, efeitos desse trabalho ainda estão sendo identificados.

Conclusões: os resultados obtidos em MS, TO e AM revelam a capacidade que a abordagem baseada na tríade ensino-aprendizagem-trabalho possui para a institucionalização de novas práticas de trabalho nos serviços de saúde.

Palavras-chave Avaliação de serviços de saúde, Atenção básica, Institucionalização
Métodos: foi desenvolvido um processo de ensinopara a organização da capacitação, para a elabo- 


\section{Introdução}

Com o movimento de descentralização da gestão do Sistema Único de Saúde (SUS), a gestão federal passou a desempenhar um papel fortemente direcionador e regulador do sistema, através de políticas públicas que determinam os movimentos e as práticas de gestão e de assistência bem como influenciam nas relações de poder ao definir campos de essencialidade e dependência. ${ }^{1}$

A década de 1990 foi praticamente toda voltada para a implementação da descentralização do sistema de saúde para o nível municipal e para a ampliação do acesso. As ações desenvolvidas no setor fortaleceram o poder local, ampliaram e qualificaram os quadros de servidores, organizaram a rede básica e avançaram na definição dos sistemas de saúde municipais.

Como unidades sociais, as organizações estão estreitamente vinculadas e reagem às diretrizes valorativas e normativas, dadas por atores externos que se inserem em seus diferentes níveis afetando a sua política e sua estrutura. ${ }^{2}$ Nesse sentido, na mesma época em que os municípios eram fortalecidos mediante políticas públicas a eles dirigidas e, diante da grande permeabilidade na dinâmica organizacional, houve fragilização das Secretarias Estaduais de Saúde (SES), que sofreram um movimento inverso, de diminuição do seu papel e enfraquecimento do poder decisório.

A normatização realizada pelo Ministério da Saúde, em particular a Norma Operacional de Assistência à Saúde (NOAS) 2002, deu às Secretarias Estaduais de Saúde a responsabilidade e a capacidade de gerir o sistema estadual por meio de instrumentos como o Plano Diretor de Regionalização (PDR) e a Programação Pactuada Integrada (PPI). Esses instrumentos fortaleceram as funções de assessoramento aos municípios e de regulação da assistência estadual e regional.

Nesse processo de redefinição dos papéis e de fortalecimento das estruturas de gestão nas três esferas de governo, o Ministério da Saúde, com o propósito de dar suporte ao processo decisório no âmbito do sistema de saúde, estabeleceu a política de institucionalização da avaliação. Essa estratégia tem como objetivo monitorar e avaliar a atenção básica instrumentalizando a gestão e fomentar a cultura avaliativa nas três instâncias de gestão do SUS. Entre os componentes dessa política destaca-se o desenvolvimento da capacidade avaliativa da atenção básica. ${ }^{3}$

O primeiro passo desse processo foi o fortalecimento do nível estadual, que teria, posteriormente, o papel de implementar o monitoramento e a avaliação da atenção básica no nível municipal com o suporte técnico-financeiro do Ministério da Saúde através do componente III do Projeto de Expansão e Consolidação da Saúde da Família (PROESF), financiado pelo Banco Internacional para Reconstrução e Desenvolvimento (BIRD). ${ }^{4}$

A implementação dessa política se deu através do estabelecimento de diretrizes e orientações 5 e da convocação dos estados para a apresentação dos planos estaduais objetivando o fortalecimento das ações de monitoramento e avaliação da atenção básica. ${ }^{4}$ Para atingir os objetivos estabelecidos, o Ministério da Saúde definiu como etapas: a) a elaboração, pela SES, de um plano estadual de fortalecimento da capacidade técnica em monitoramento e avaliação da atenção básica (MA da $\mathrm{AB}$ ); b) a capacitação da equipe estadual e a elaboração de uma proposta metodológica de MA da AB para o Estado; c) a execução das ações previstas na proposta metodológica; e, finalmente, d) a disseminação da prática da avaliação da atenção básica na esfera dos municípios do Estado.

A inclusão do monitoramento e avaliação da atenção básica como exigência para a aprovação dos planos estaduais visava tanto discutir diretrizes para a promoção de uma cultura avaliativa quanto estabelecer pactos para os aspectos mais importantes da atenção básica a serem monitorados e avaliados por meio da mobilização de técnicos, gestores e pesquisadores. A proposta metodológica de monitoramento e avaliação da atenção básica (PM de MA da $\mathrm{AB}$ ) envolvia todos os atores e procurava redefinir papéis, responsabilidades e investimentos. 6

Para a implementação do plano de fortalecimento do MA da AB, o Ministério da Saúde estabeleceu como requisito a contratação de centros colaboradores, unidades ou grupos de instituições de ensino superior com conhecimento e experiência em avaliação, para realizar o processo de capacitação e assessorar a elaboração da $\mathrm{PM}$ de $\mathrm{MA}$ da $\mathrm{AB}$. O centro colaborador em avaliação do Departamento de Saúde Coletiva da Universidade Federal de Mato Grosso do Sul foi contratado em 2005 para assessorar os Estados de Mato Grosso do Sul e do Tocantins e em 2006 para o Estado do Amazonas.

\section{Métodos}

Institucionalização, no campo organizacional, significa "processo de transformar crenças e ações em regras de conduta social" (Fonseca, 2003: 58):7

[...] por influência de mecanismos de aceitação e repro- 
dução, tais regras tornam-se padrões e passam a ser encaradas como rotinas naturais, ou concepções amplamente compartilhadas da realidade.

Para o centro colaborador a institucionalização de práticas de monitoramento e avaliação como um dos produtos esperados do trabalho a ser desenvolvido implicou em desenvolver na equipe das SES que atuava na atenção básica autonomia e auto-organização sobre todo o seu processo de trabalho. A rigor, considera-se um agente autônomo "quando suas ações são verdadeiramente suas". ${ }^{8}$ No campo das organizações, a autonomia, ao invés de excluir, traz em si a idéia de interdependência. ${ }^{9} \mathrm{Na}$ saúde, a autonomia não é vista sob o ponto de vista do isolamento mas sim como o preparo, a aptidão das pessoas para desenvolver suas atividades e resolver problemas na sua área de atuação. 10

Este propósito implicou enormes desafios porque a autonomia só se viabiliza quando os indivíduos orientam suas ações com base em sua própria capacidade de julgamento. Para isso, os serviços devem ser preferencialmente auto-organizados, ou seja, a organização deve favorecer a identificação e criação de fluxos e conexões multidirecionais capazes de ampliar e agilizar a comunicação entre atividades e setores. 11,12

Com isso, foi possível organizar um plano de assessoria tendo como referencial as teorias de planejamento estratégico desenvolvidas por Mário Testa, associadas a métodos pedagógicos reflexivos e problematizadores. Ambos os pressupostos são coerentes e complementares entre si, uma vez que o planejamento propicia análises situacionais $\mathrm{e}$ mudanças organizacionais e as metodologias ativas focam as mudanças no conhecimento/práticas dos sujeitos/agentes.

Para Testa, 13,14 as mudanças acontecem quando o processo viabiliza três momentos, os quais ele nomina: a) de abertura, capaz de mobilizar, questionar o existente e causar desconfortos em relação às práticas e ao conhecimento utilizado; b) de avanço, que implanta a mudança propriamente dita $\mathrm{e}$ discute novos papéis, novos conhecimentos e modos de fazer; c) de consolidação, que prevê retrocessos e admite novas estratégias de modo a assegurar as mudanças. A partir deste pressuposto teórico em planejamento, as metodologias selecionadas para as atividades partiram da premissa de que o aprendizado é um processo complexo e ocorre de forma não linear. O aprendizado se estrutura a partir das associações e conexões que cada sujeito faz, ou seja, para que a aprendizagem produza mudanças ela precisa ser significativa. 15
Cyrino e Toralles-Pereira15 afirmam que o ensino de novos conteúdos deve desafiar os sujeitos a avançar em seus conhecimentos:

O conteúdo novo deve apoiar-se numa estrutura cognitiva já existente, o que exige [...] verificar o que o aluno sabe, para, de um lado, relacionar os novos conteúdos à experiência do aluno - a continuidade - e de outro, provocar novas necessidades e desafios pela análise crítica, levando o aluno a ultrapassar a sua experiência [...].

\section{Organização do processo de ensino- aprendizagem-trabalho}

Em função do prazo estabelecido pelo Ministério da Saúde para realizar a capacitação do corpo técnico da SES e a elaboração de uma PM de MA da AB foi necessário associar o processo de capacitação às atividades de elaboração da proposta metodológica, concebendo e implementando um processo com o duplo objetivo de qualificar e produzir um trabalho concreto. A estratégia de institucionalização do MA da $\mathrm{AB}$ adotada pelo centro colaborador foi o de propiciar ao grupo: incorporação de referencial teóricometodológico básico, realização de exercício de concepção e estruturação de processos avaliativos, execução de atividade prática real de planejamento e programação em saúde e fornecimento de capacidade de intervir no ambiente organizacional que permitisse implementar as propostas feitas. Para atingir esses objetivos foi estruturado um processo de ensino-aprendizagem-trabalho através da realização de dois cursos de capacitação: um de capacitação em monitoramento e avaliação de programas e serviços de saúde, de 120 horas, e outro em técnicas de organização e racionalização administrativa de 60 horas.

\section{Princípios e critérios para a organização do processo de ensino-aprendizagem-trabalho}

A institucionalização da avaliação implicava na incorporação da avaliação na prática dos serviços. Esse objetivo só seria atingido se os sistemas, métodos e procedimentos fossem não somente concebidos, mas também implementados e executados de forma rotineira e permanente. Para atingir esse objetivo um conjunto de princípios e critérios foi estabelecido para organizar o processo de ensinoaprendizagem-trabalho a ser implementado:

a) Os participantes do grupo deveriam ser servidores efetivos pertencentes ao quadro permanente da Secretaria Estadual de Saúde a fim de buscar resultados os mais duradouros possíveis para o 
serviço.

b) As questões abordadas no processo de formação seriam aquelas identificadas e vivenciadas pelos participantes do curso, a fim de garantir a aderência dos temas à realidade do Estado.

c) Os produtos das atividades de formação deveriam trazer respostas às questões e necessidades trazidas pelos participantes do curso, de forma a exercer uma função motivadora no aperfeiçoamento da sua prática e na solução dos seus problemas.

d) As propostas feitas no curso deveriam ser factíveis e ter a possibilidade de continuidade de forma autônoma pelos participantes, respeitando: (i) o nível teórico-conceitual alcançado pelo grupo; (ii) a capacidade de trabalho e disponibilidade do grupo para desenvolver as atividades de MA da AB e; (iii) as limitações e obstáculos existentes na organização do serviço que o participante não tivesse governabilidade de ultrapassálos ou removê-los.

e) Os conhecimentos e habilidades a serem fornecidos na capacitação através de métodos ativos, participativos e problematizadores deveriam capacitá-los não somente para conceber e organizar processos avaliativos, mas também para atuar na organização de serviços de saúde a fim de viabilizar a implementação das propostas produzidas.

f) A criação de um ambiente favorável para a implementação das propostas de MA da AB no âmbito da SES deveria ser buscada através do envolvimento de todos os setores da SES que atuassem ou que tivessem interface com o monitoramento e avaliação da atenção básica e da criação de vínculos entre os participantes através da interação, integração e produção coletiva.

Princípios e critérios para a elaboração da proposta metodológica de monitoramento e avaliação da atenção básica (PM de MA da AB)

A elaboração de uma PM de MA da AB, definida pelo Ministério da Saúde 5 como: "o desenho estratégico e metodológico da SES para o fortalecimento das ações de monitoramento e avaliação da atenção básica contemplando os métodos, abordagens, ações prioritárias, atividades, metas, indicadores e instrumentos que serão utilizados, além dos custos e investimentos pensados para melhor estruturação das ações de monitoramento e avaliação da atenção básica da SES”, implicava, na prática, na realização de um processo de planejamento e programação.
Considerando que a $\mathrm{PM}$ de $\mathrm{MA}$ da $\mathrm{AB}$ seria construída no interior de um processo de capacitação, foi necessário estabelecer um segundo conjunto de princípios e critérios que permitisse assegurar a qualidade necessária para um trabalho dessa natureza:

a) Em função dos prazos existentes, não seria possível realizar um processo tradicional de planejamento que se inicia com a elaboração de um diagnóstico em saúde analisando diretamente a situação do Estado. Nessas condições a estratégia utilizada para obter o melhor diagnóstico possível foi a de garantir a presença daqueles que melhor conheciam a realidade do estado na elaboração do trabalho a ser desenvolvido, ou seja, os próprios técnicos das unidades da SES.

b) Para o diagnóstico a ser realizado sobre a situação do MA da $\mathrm{AB}$ era fundamental que todas as áreas da SES que participassem diretamente ou tivessem interface com a $\mathrm{AB}$ estivessem representadas. Para atingir esse objetivo, a escolha dos participantes constituiu uma atividade crítica, pois o conhecimento da realidade existente no Estado dependia do conhecimento trazido pelos participantes da capacitação; logo, essa representatividade foi um dos critérios para a seleção dos participantes do grupo.

c) A factibilidade da implantação das propostas foi considerada prioritária em relação a um esforço de busca de maior qualidade técnica, pois havia o risco de comprometer a obtenção do produto no prazo existente e de elaborar propostas com poucas possibilidades de serem implementadas. Nessas condições, a qualidade final do trabalho foi pautada no conhecimento apropriado pelos participantes, nas limitações estruturais da SES e na capacidade de trabalho do grupo.

d) Considerando que a qualidade do trabalho dependia da assimilação de novos conhecimentos teóricos pelos participantes do grupo, as sessões eram iniciadas com revisões e novos aportes teóricos através de aulas, leituras de textos, discussões e reflexões sobre o tema abordado. Visou-se, dessa forma, o aumento do conhecimento teórico do grupo sobre monitoramento e avaliação, de forma a refletir na melhoria da qualidade das propostas que fossem elaboradas.

e) A expansão e consolidação da atenção básica constituíam o objetivo maior do Ministério da Saúde, considerou-se que para isso era necessário monitorar não somente as ações já instituídas (PPI, PDR, Pacto da AB, entre outros) mas também todos os outros fatores e problemas que estariam afetando essa expansão no Estado. Para 
avançar seria necessário inovar e isso só seria obtido caso se abordasse aspectos ainda não explorados nas atividades que já vinham sendo realizadas pela SES.

\section{Procedimentos didático-pedagógicos utilizados} no processo de ensino-aprendizagem-trabalho

A abordagem, os princípios e critérios adotados pela assessoria faziam com que as atividades desenvolvidas buscassem permanentemente: o envolvimento e comprometimento dos participantes nas atividades da capacitação; a motivação de identificar e buscar respostas aos seus problemas; a valorização dos produtos elaborados; e a criação de um compromisso com a implementação de um produto que era resultado do seu próprio trabalho. Essa postura formativa era necessária, pois além de capacitar recursos humanos, era necessário produzir uma PM de MA da $\mathrm{AB}$ e institucionalizar uma prática de avaliação nos serviços de saúde.

a) Considerando a objetividade necessária para atingir os objetivos da capacitação-trabalho a ser desenvolvida, os conteúdos teóricos, em termos de abrangência, volume e profundidade, foram estabelecidos visando proporcionar o conhecimento necessário para o desenvolvimento de sistemas e a implementação de processos avaliativos, sempre respeitando o nível de conhecimento prévio já existente no grupo.

b) Como a elaboração de uma PM de MA da AB implicava a realização de um processo de planejamento e programação, o eixo norteador da escolha dos conteúdos e da definição da sequência de temas abordados foi tipicamente o de um processo de planejamento em saúde.

c) Considerando que a unidade de produção de conhecimento-trabalho era uma atividade coletiva realizada em grupos (oficina de trabalho), a composição de cada grupo foi cuidadosamente estabelecida em função dos objetivos e dos produtos a serem obtidos e do conhecimento prático e técnico de cada participante.

d) A metodologia utilizada para a capacitação foi baseada em processos participativos ativos e problematizadores, considerados os mais adequados para buscar o envolvimento e a interação de todos os participantes, para motivá-los à produção coletiva, para criar uma identidade com as propostas por eles desenvolvidas e obter um comprometimento para a sua implantação.

e) A dinâmica utilizada para a condução das sessões foi ajustada para cada sessão, no entanto, ela se constituía, de forma geral, de um aporte de conhecimentos teóricos sobre o tema e sobre o trabalho a ser desenvolvido, de estímulos às reflexões teoria-prática pelos participantes, de orientações para o desenvolvimento das atividades, de facilitação das interações intra e intergrupais, e de orientações para a elaboração das sistematizações e sínteses finais. Essa forma de condução da sessão tinha, entre outros, o objetivo de garantir coerência e consistência dos produtos obtidos na sessão, uma vez que eles se constituiriam em elementos da PM de MA da AB.

f) Os cursos foram subdivididos em módulos a fim de que houvesse momentos de concentração, com o objetivo central de fornecer aportes teóricos e práticos para a elevação do nível de conhecimento e de habilidades dos participantes, e de momentos de dispersão, que eram destinados fundamentalmente para a aplicação dos conhecimentos e habilidades adquiridos na produção dos elementos que constituiriam a PM de MA da AB.

\section{Resultados}

As atividades desenvolvidas nos Estados de Mato Grosso do Sul (MS) e Tocantins (TO) em 2006 e no Estado do Amazonas (AM) em 2007 foram realizadas em três etapas: na primeira, foi realizado o primeiro módulo do curso de avaliação da atenção básica, no qual foram fornecidos conhecimentos conceituais e teóricos seguidos de uma aplicação prática desses conhecimentos sobre a realidade do Estado. Resultou um diagnóstico preliminar da situação da $\mathrm{AB}$ e dos mecanismos de monitoramento e avaliação existentes no Estado, permitindo a elaboração de uma PM de MA da $\mathrm{AB}$ preliminar que foi, em seguida, submetida à apreciação das respectivas SES para fins de aperfeiçoamento e validação.

A segunda etapa foi constituída pela realização do curso de técnicas de organização e racionalização administrativa e do segundo módulo do curso de avaliação da atenção básica, onde foram aprofundados os conhecimentos referentes à construção de indicadores, de parâmetros e de planos de avaliação. $\mathrm{O}$ resultado desse conjunto de atividades foi a elaboração de um diagnóstico detalhado do $\mathrm{MA}$ da $\mathrm{AB}$ no Estado que permitiu construir uma anteproposta que foi analisada, discutida, detalhada, complementada com a programação das atividades e, finalmente, validada pela SES no último módulo do curso.

Os trabalhos desenvolvidos nos Estados de MS e TO tiveram a duração de um ano e no AM de sete meses. No Amazonas, devido ao prazo mais curto para a execução das atividades, foi realizado apenas um curso, o de avaliação da atenção básica, mas 
incorporando os conteúdos principais do curso de técnicas de organização e racionalização administrativa.

\section{O desenvolvimento das atividades em MS, TO e AM}

A inserção do órgão responsável pela atenção básica na estrutura da SES do Estado, as suas atribuições e competências eram diferentes entre os Estados. Em MS esse órgão era responsável pelo Programa de Agentes Comunitários de Saúde / Programa Saúde da Família (PACS/PSF) e as demais atividades da atenção básica eram distri-buídas nas várias coordenações de programas de saúde. Situação semelhante era encontrada no Amazonas, onde as atividades da atenção básica estavam distribuídas entre várias unidades da SES e, inclusive, entre as Fundações de Saúde do Estado. Somente no Tocantins o órgão responsável pela atenção básica reunia a gestão de todos os programas da atenção básica. Apesar de essa situação refletir na autonomia das coordenações estaduais para compor um grupo representativo de todas as unidades envolvidas com a atenção básica, foi possível constituir nos três estados grupos com essas características. Essa atividade era crítica, pois do seu sucesso dependia a representatividade da situação da atenção básica e a sua aderência à realidade do Estado.

Nos Estados de TO e AM os módulos foram realizados em uma semana, enquanto em MS os módulos foram subdivididos em sessões de dois dias, com duas semanas de intervalo entre elas. As atividades realizadas nos três Estados permitiram desenvolver PM de MA da AB próprias para cada um e com encaminhamentos distintos em função das realidades existentes e vivenciadas pelos seus responsáveis.

\section{Resultados em Mato Grosso do Sul}

A PM de MA da AB de Mato Grosso do Sul estabeleceu duas vertentes de trabalho: o acompanhamento de um conjunto de indicadores prioritários para a atenção básica ao nível central e a implementação experimental do MA da $\mathrm{AB}$ ao nível municipal. Os indicadores escolhidos pela equipe técnica se situavam nas diversas áreas da atenção básica, tendo sido estabelecidos parâmetros, fórmulas e os sistemas de informação a serem utilizados.

Para a implementação experimental foram constituídas equipes com técnicos egressos da capacitação realizada pelo centro colaborador para atuar nos quatro municípios selecionados: Campo Grande e Dourados, de grande porte, Três Lagoas, município de porte médio e Água Clara, de pequeno porte. A partir desse trabalho, muitos avanços foram observados no estado: integração entre as áreas técnicas; criação dos grupos municipais e regionais de monitoramento e avaliação; criação da coordenadoria estadual de atenção básica; criação da gerência dos indicadores da atenção básica e de monitoramento e avaliação e o comprometimento do gestor estadual com a atenção básica. Segundo a coordenação estadual de atenção básica a implantação nos quatro municípios foi iniciada. No entanto, alguns desafios ainda serão enfrentados e será necessário levar em conta a "continuidade ao processo; a necessidade de ampliar a cobertura (disseminar a proposta); o fortalecimento dos grupos estaduais, regionais e municipais; a implantação da avaliação da melhoria da qualidade (AMQ) da atenção básica".16

\section{Resultados no Tocantins}

A PM de MA da AB do Tocantins também estabeleceu duas vertentes de trabalho: uma consistia na realização do monitoramento de 15 indicadores dos principais programas de saúde da atenção básica pelo nível central da SES-TO e outra que consistiu na implementação experimental do $\mathrm{MA}$ da $\mathrm{AB}$ em um módulo assistencial formado por 12 municípios do Estado. Essa região foi escolhida pela facilidade de acesso e proximidade da capital. Os municípios de Palmas e Araguaína também foram incluídos por serem de grande porte.

Ainda durante a assessoria do centro colaborador, foi priorizada a atividade de monitoramento dos indicadores da $\mathrm{AB}$ pelo nível central de SES que resultou no desenvolvimento de instrumentos de monitoramento para os principais programas de saúde da atenção básica do Estado. Com esse trabalho foi possível: a) agilizar os fluxos de informações no interior da Secretaria; b) desenvolver, para cada indicador, classes e parâmetros aceitáveis para cada conjunto de municípios classificados conforme ocorrência de agravos; c) elaborar instrumentos gráficos ou numéricos com indicações avaliativas (situações não aceitáveis, aceitáveis, razoáveis ou ótimas). Ficou estabelecido, também, que indicadores não aceitáveis deveriam desencadear processos avaliativos sobre os motivos da sua ocorrência.

As atividades de MA da AB tiveram continuidade no Estado uma vez que, quase dois anos após o término da assessoria do centro colaborador, o grupo técnico da SES apresentou na III Mostra Nacional de Produção em Saúde da Família o trabalho intitulado 
"A institucionalização do monitoramento e avaliação da atenção básica, um caminho para a qualidade". 17 Nesse evento é citada a criação de uma gerência de monitoramento e avaliação e a realização de cursos de capacitação em avaliação, e é fornecida a informação de que, em 2007, 50\% dos municípios do Estado estavam sendo monitorados.

\section{Resultados no Amazonas}

As peculiaridades da atenção básica no Amazonas, a fragmentação e baixa confiabilidade nos sistemas de informações e a demora para a alimentação dos bancos de dados fez com que o grupo encaminhasse as atividades de MA da $\mathrm{AB}$ em duas frentes: uma referia-se ao acompanhamento dos indicadores propriamente ditos e outra dizia respeito à melhoria dos fluxos e da confiabilidade das informações.

Foram selecionadas seis áreas técnicas para serem monitoradas e avaliadas por meio do acompanhamento de dez indicadores. Em seis meses foi possível traçar um panorama dos indicadores selecionados, discutir e estratificar as situações dos mesmos em todos os municípios do Estado e tecer metas plausíveis para o ano de 2007-2008, tanto para melhorar os indicadores quanto melhorar os fluxos e a qualidade dos sistemas de informações. Concomitante a essas atividades, o grupo coordenador da atenção básica manteve a assessoria aos municípios para as demais fases da Avaliação para a Melhoria da Qualidade (AMQ), sistema implementado pelo Ministério da Saúde.

A qualidade e riqueza dos produtos que foram desenvolvidos pelo grupo coordenador da atenção básica no tempo disponível foram surpreendentes em face das dificuldades e limitações vivenciadas pelos técnicos no Estado. Em 2008, na mesma mesa redonda da III Mostra Nacional de Produção em Saúde da Família onde foi apresentado o trabalho do Tocantins, a SES-AM apresentou o trabalho "Integrando as ações de monitoramento e avaliação da atenção da básica com implantação da AMQ no Amazonas". ${ }^{17}$ Para a equipe, a integração, motivação e melhoria da capacidade técnica das equipes da SES e da Secretaria Municipal de Saúde de Manaus, juntamente com o apoio do Ministério da Saúde, centro colaborador Universidade Federal do Mato Grosso do Sul (UFMS) e Secretarias Munici-pais de Saúde, superou entraves, como por exemplo, a fragmentação de ações da atenção básica dentro da SES$\mathrm{AM}$ e em fundações a ela subordinadas, favorecendo a agregação do conhecimento e das informa-ções diluídas nas diversas instituições. Tais mudanças melhoraram a dinâmica operacional da atenção básica na Secretaria.

\section{Considerações finais}

Os resultados obtidos nos estados de MS, TO e AM revelam a capacidade que a abordagem baseada na tríade ensino-aprendizagem-trabalho possui para a incorporação de novos conhecimentos e de novos procedimentos nas práticas de trabalho dos participantes desse processo de capacitação. A utilização de problemas concretos trazidos pelos participantes da capacitação garantiu a aderência da formação e do trabalho produzido à realidade dos serviços assim como o envolvimento e o engajamento dos responsáveis pelos produtos obtidos na implementação ou concretização das propostas feitas.

A avaliação realizada pelos participantes dos processos de ensino-aprendizagem-trabalho desenvolvidos nos três Estados descreveu as aquisições em termos de conhecimento e prática decorrentes do processo de formação. No entanto, o objetivo maior de todo esse processo, que é o de institucionalizar o MA da $\mathrm{AB}$ através da disseminação e implementação de processos e mecanismos de monitoramento e avaliação no interior da própria SES, nos municípios e nos serviços de saúde, ainda é frágil e vai depender de uma ação continuada e sustentada do Ministério da Saúde.

As dificuldades inerentes a esse processo foram levadas em consideração pelo Ministério da Saúde, pois foi facultada, a critério dos Estados, a utilização de recursos da segunda fase no componente III do PROESF para dar continuidade ao assessoramento dos centros colaboradores. Isto visava consolidar as mudanças obtidas e implementar as propostas metodológicas realizadas, cujo foco seria, neste segundo momento, a disseminação da cultura avaliativa para o nível municipal.

Após mais de dois anos sem que fosse dada continuidade ao financiamento do PROESF, pôde-se observar que o processo de intervenção adotado pelo centro colaborador obteve uma eficácia suficiente para garantir que ações de $\mathrm{MA}$ da $\mathrm{AB}$ tivessem continuidade no âmbito das SES, indicando claramente a incorporação, embora limitada aos técnicos que participaram do processo de formação, de uma capacidade técnica, mas também, e talvez mais importante, de uma preocupação e de uma postura voltada para a avaliação das ações em saúde, conforme pode ser observado nos trabalhos apresentados após o término das assessorias prestadas aos Estados.

Os resultados observados indicam o potencial que técnicas participativas de ensino-aprendizagem- 
trabalho, ancoradas no conhecimento e experiência dos participantes, na utilização de problemas reais vivenciados por eles e na produção de propostas

\section{Referências}

1. Thompson JD. Dinâmica organizacional: fundamentos sociológicos da teoria administrativa. São Paulo: McGraw Hill; 1976.

2. Vieira MMF, Carvalho CA. Organizações, instituições e poder no Brasil. Rio de Janeiro: FGV; 2003.

3. Brasil. Ministério da Saúde. Proesf. Matriz de programação POA 2006. [on line]. Brasília, DF; 2006. [acesso 24 mar 2009]. Disponível em: http://dtr2002.saude.gov.br/proesf/

4. Brasil. Ministério da Saúde. Planos estaduais para o fortalecimento das ações de monitoramento e avaliação da atenção básica: diretrizes e orientações. Brasília, DF; 2004.

5. Brasil. Ministério da Saúde. Portaria $n^{\circ} 588 / \mathrm{GM}, 7$ de abril de 2004, que aprova Convocatória Pública $n^{\circ}$ 01/2004 para que Unidades Federadas (UF) apresentem planos estaduais para o fortalecimento das ações de monitoramento e avaliação da atenção básica no âmbito do Projeto de Expansão e Consolidação do Saúde da Família - PROESF. [on line]. Brasília; 2004. [acesso 19 mar 2009]. Disponível em: http://dtr2001.saude.gov.br/sas/

6. Felisberto E. Monitoramento e avaliação na atenção básica: novos horizontes. Rev Bras Saúde Matern Infant. 2004; 4: 317-21.

7. Fonseca VS. A abordagem institucional nos estudos organizacionais: bases conceituais e desenvolvimentos contemporâneos. In: Vieira MMF, Carvalho CA, organizadores. Organizações, instituições e poder no Brasil. Rio de Janeiro: FGV; 2003. p. 45-66.

8. Blackburn S. Dicionário Oxford de filosofia. Rio de Janeiro: Ed. Jorge Zahar; 1997.

9. Habermas J. Constitutional democracy: a paradoxical union of contradictory principles? In: Political theory. [periódico on line]. $2001 \mathrm{dez}$ [acesso 3 mar 09]; 29 (dec): 766-81. Disponível em: http://online.sagepub.com

Recebido em 17 de maio de 2010

Versão final apresentada em 6 de agosto de 2010

Aprovado em 24 de agosto de 2010 concretas de soluções dos problemas existentes, têm para a implementação e institucionalização da avaliação nos serviços de saúde.

10. Cecílio LCO. Autonomia versus controle dos trabalhadores: a gestão do poder no hospital. Ciênc Saúde Coletiva. 1999; 4: 315-29.

11. Agostinho M. A organização emergente: gerenciando o processo de auto-organização [tese]. Rio de Janeiro: Universidade Federal do Rio de Janeiro; 2001.

12. McMaster MD. The intelligence advantage: organizing for complexity. Boston: Butterworth-Heinemann; 1996.

13. Testa M. Pensamento estratégico e lógica de programação: o caso da saúde. São Paulo: Hucitec; 1995.

14. Testa M. Pensar em saúde. Porto Alegre: Artes médicas/ABRASCO; 1992.

15. Cyrino EG, Torales-Pereira ML. Trabalhando com estratégias de ensino-aprendizado por descoberta na área da saúde: a problematização e a aprendizagem baseada em problemas. Cad Saúde Pública. 2004; 20: 780-8.

16. Tobal AMM. Expansão com qualidade e valorização dos resultados. In: Anais do $3^{\circ}$ Seminário Internacional de Atenção Primária/Saúde da Família; 2007 dez 13-15; Recife, PE, BR [acesso 2009 mar 19]. Disponível em: http://dtr2004.saude.gov.br/dab/docs/eventos/seminario_int er_dab/adriana_tobal_bra.pdf.

17. Anais da III Mostra Nacional de Produção em Saúde da Família; 2008 ago 5-8; Brasília, DF: Ministério da Saúde; 2008. [acesso 2009 mar 19]. Disponível em http://dtr2004. saude.gov.br/dab/evento/mostra/documentos/mr.pdf. 